\title{
Linx
}

Revue des linguistes de l'université Paris X Nanterre

$66-67 \mid 2012$

Dynamiques de la construction des sens attendus et inattendus dans les langues

\section{Présentation : dynamiques de la construction des sens attendus et inattendus dans les langues}

Martine Sekali et Anne Trévise

\section{(2) OpenEdition}

\section{Journals}

Édition électronique

URL : http://journals.openedition.org/linx/1441

DOI : 10.4000/linx.1441

ISSN : 2118-9692

Éditeur

Presses universitaires de Paris Nanterre

Édition imprimée

Date de publication : 15 septembre 2012

Pagination : $9-12$

ISSN : 0246-8743

Référence électronique

Martine Sekali et Anne Trévise, "Présentation : dynamiques de la construction des sens attendus et inattendus dans les langues », Linx [En ligne], 66-67 | 2012, mis en ligne le 15 septembre 2013,

consulté le 22 septembre 2020. URL : http://journals.openedition.org/linx/1441 ; DOI : https://doi.org/ $10.4000 / \operatorname{linx} .1441$ 


\title{
Présentation : dynamiques de la construction des sens attendus et inattendus dans les langues
}

\author{
Martine Sekali et Anne Trévise*
}

\section{Présentation}

Le présent numéro thématique rassemble huit articles qui visent à préciser l'étude de la construction/reconstruction d'un sens «attendu » vs « inattendu $»^{1}:$ dans les textes, certaines représentations sont repérées comme saillantes, non attendues, contre-attendues, exploratrices de frontières notionnelles ou définies comme polémiques et associées à une modalité de discordance inter-sujets.

Est ainsi visée une définition plus précise de ces «itinéraires bis » du sens, en tant que mode spécifique de prise en charge intersubjective du dire : le travail cherche à inclure notamment les processus linguistiques d'opposition, de concession, de

\footnotetext{
* sekali@,u-paris10.fr ; anne.trevise@,wanadoo.fr

${ }^{1}$ Ces articles sont issus de contributions au colloque international de linguistique organisé par le groupe de recherche GReG les 25 et 26 janvier 2013, intitulé : «Sens attendu/inattendu dans les langues ». Ce colloque se situait dans la continuité d'une analyse épistémologique du paramétrage linguistique du sens dans les systèmes de langues, et faisait suite à deux autres colloques sur le même thème. Le groupe GReG (Groupe de Réflexion sur les Grammaires) est rattaché au centre de recherche CREA (EA 370) de l'université Paris Ouest-Nanterre-la Défense (http://anglais.uparis10.fr/spip.php?rubrique55).
} 
ré-assertion, de contre-orientation, de heurts par rapport à une norme attendue. On envisagera d'étudier la nature de cette zone de sens intermédiaire construite ou prescrite, ainsi que les phénomènes linguistiques responsables de sa présence et de son élaboration/déviation. L'approche multidimensionnelle de ces interfaces dynamiques liées à la (re)construction du sens se veut résolument linguistique et s'appuie sur des corpus de faits de langues authentiques.

Après un article présenté par Anne Trévise et Martine Sekali (Université Paris Ouest Nanterre la Défense), qui précise dans une première partie introductive les enjeux épistémologiques d'une telle problématique, le numéro s'articule autour de trois mouvements permettant un éclairage sur différentes composantes de ces phénomènes :

\section{II. l'in/attendu dans les dynamiques binaires et ternaires}

Il s'agit d'une étude de la construction d'implicites dans les relations interpropositionnelles, d'inférences logiques souvent valuées impliquant des pré-construits élaborés dans et par la langue, en rapport avec des présupposés extralinguistiques (topoi) qui dépendent de la connaissance culturelle du monde.

Dans cette partie, Agnès Leroux (Université Paris Ouest Nanterre la Défense) propose une étude contrastive de l'adverbe anglais even et de ses multiples traductions en français, dans le cadre de la Théorie des Opérations Prédicatives et Enonciatives. Even est analysé en tant qu'il marque un hiatus entre une situation préalable, définie par l'adverbe comme attendue par l'énonciateur origine, et une situation effective. Dans la souplesse du jeu sur ce passage de frontière (entre l'intérieur et l'extérieur du domaine notionnel), le choix de la traduction en français par encore plus, presque, même ou voire est mis en rapport par l'auteur avec une mise en relief d'un des paramètres contextuels de construction de la référence à l'inattendu au détriment des autres. Dans l'article de Stéphane Gresset (Université Paris Diderot), la relation entre concession et construction d'un sens in/attendu est considérée cette fois dans le cadre de la TOPE par l'analyse d'une variété d'énoncés à schéma concessif incluant tous le modal anglais may, au sein d'un discours de B. Obama. L'auteur y formalise les traits distinctifs d'une 'variation dans l'expression de la concession', à partir de l'association des schémas opératoires de may et de but, as et while, ainsi qu'à titre de comparaison, de al/ though, yet et however. L'attendu comme l'inattendu sont analysés en tant que préconstruits dans et par la langue, dans un schéma d'anti-causalité', et de balancement balancement rhétorique et intersubjectif. Zsófia Várkonyi (Université d’Orléans) apporte à ce numéro un point de vue différent sur la construction, par ce que l'auteur nomme des 'articulateurs complexes', d'un sens intermédiaire défini comme attendu ou inattendu. L'article analyse la qualification des implicites linguistiques dans les structures « if $X$, (then) $Y$, but if $Z$ (then) $T$ » et « $Y$, but if $Z$, (then) $T$ », à partir d'exemples authentiques tirés des discours oraux de Barack Obama. L'étude, ancrée dans une démarche ducrotienne inspirée de l'Argumentation dans La Langue, propose une formalisation de ces structures en termes de présupposés argumentatifs (et non logiques), et de contraintes topiques, dans une description des contraintes que ces unités 
linguistiques complexes instruisent sur la manière dont certains topoï doivent être appréhendés. Enfin Maria Svensson (Université d'Uppsala, Suède), compare le marqueur complexe même si avec le marqueur simple si moins attendu dans son emploi concessif. L'auteur propose une analyse quantitative et qualitative de données issues du corpus large C-ParaFraSe-HumSam. Les résultats de cette étude révèlent des différences formelles et sémantiques, qui sont formalisées dans le cadre de la Théorie Modale de la Polyphonie appliquée aux relations de concession, en tant que différents modes de rejet d'une inférence attendue par un être de discours autre que celui qui modalise la structure complexe.

\section{III. l'in/attendu dans les relations intersubjectives}

Toutes ces constructions binaires ou ternaires multidimensionnelles, se jouent dans l'intersubjectivité de l'activité langagière, qui gère les discordances prévues, devancées, rectifiées, manipulées, renversées, et tous ces jeux de repérages entre énonciateur et co-énonciateur, ces ajustements de tous ordres ;

Outi Duvallon (INALCO) et Réa Peltola (Université de Normandie), se référant à la TOPE, analysent les propriétés sémantiques de deux formes finnoises, le mode jussif en $-k O O n$ et le marqueur discursif $-h A n$, qui produisent des effets de sens similaires dans des contextes concessifs. Avec le jussif, le locuteur se place sur la scène énonciative comme un participant ayant un certain contrôle sur la situation alors qu'avec - $h A n$, son rôle est réduit à celui de valideur modal d'intentions qui ne sont pas les siennes. Les deux formes marquent deux mécanismes différents, l'un ayant trait à l'actualisation du procès, l'autre à la détermination de l'origine énonciative. Dans un deuxième temps sont étudiées des relations concessives ou causales auxquelles les énoncés jussifs et les énoncés en - $h A n$ participent dans le jeu des attentes et des réorientations de discours. Tatiana Bottineau (INALCO) aborde l'opposition «attendu»/《inattendu» dans l'étude de l'intersubjectivité construite par les quantifieurs russes «N raz » et «N-ždy » en position thématique. Son étude s'appuie sur des critères formels et tient compte de paramètres énonciatifs tels que l'adéquation à une pré-construction valuée d'altérité de valeurs, les coordonnées énonciatives et le concept de visée. Les deux quantifieurs ont un emploi neutre lorsque leur portée propose l'explicitation du contenu explicité et qu'elle s'inscrit dans une relation de

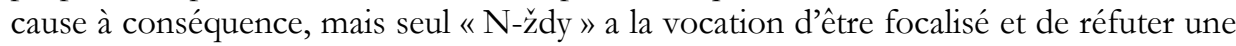
attente préconstruite ou de construire des valeurs concessives ou polémiques.

\section{IV. l'inattendu métalinguistique}

La notion d'«inattendu» est aussi apparue à un niveau purement métalinguistique: pourquoi deux formes, par exemple deux prépositions, de sens apparemment proche, revêtent, suivant les contextes, des valeurs contraires et vice versa.

Lise Hamelin (Université de Cergy Pontoise), dans le cadre de la Théorie des Opérations Prédicatives et Énonciatives, propose une modélisation du fonctionnement 
des prépositions out of et from en anglais qui lui permet de prédire et de circonscrire les zones de convergence, de divergence et donc de concurrence éventuelle entre ces deux marqueurs. Plus précisément, elle représente ces marqueurs au moyen du système ternaire des opérations de repérage de la TOPE (identification, différenciation, rupture), afin de faire émerger les caractéristiques des zones de concurrence entre les deux prépositions.

Les dynamiques de la construction des sens attendus et inattendus dans différentes langues (français, anglais, russe et finnois), sont ainsi précisées par le concours/la concurrence d'approches théoriques différentes (Théorie des Opérations Énonciatives et Prédicatives, Argumentation dans La Langue, Théorie Modale de la Polyphonie, cadre guillaumien). 